\title{
Visceral-to-Subcutaneous Abdominal Fat Ratio Is Associated with Nonalcoholic Fatty Liver Disease and Liver Fibrosis
}

\author{
Chan-Hee Jung ${ }^{1, *}$, Eun-Jung Rhee ${ }^{2, *}$, Hyemi Kwon², Yoosoo Chang ${ }^{3,4,5}$, Seungho Ryu ${ }^{3,4,5}$, Won-Young Lee ${ }^{2}$ \\ ${ }^{1}$ Division of Endocrinology and Metabolism, Department of Internal Medicine, Soonchunhyang University Bucheon Hospital, \\ Soonchunhyang University College of Medicine, Bucheon; ${ }^{2}$ Division of Endocrinology and Metabolism, Department of Internal \\ Medicine, ${ }^{3}$ Department of Occupational and Environmental Medicine, ${ }^{4}$ Center for Cohort Studies, Total Healthcare Center, \\ Kangbuk Samsung Hospital, Sungkyunkwan University School of Medicine; ${ }^{5}$ Department of Clinical Research Design \& \\ Evaluation, Samsung Advanced Institute for Health Sciences \& Technology, Sungkyunkwan University, Seoul, Korea
}

Background: We evaluated the association of visceral-to-subcutaneous fat ratio (VSR) with nonalcoholic fatty liver disease (NAFLD) and advanced fibrosis degree based on noninvasive serum fibrosis markers in the general population with NAFLD.

Methods: This is a cross-sectional study, in 7,465 Korean adults who underwent health screening examinations. NAFLD was defined as fatty liver detected on ultrasonography, and visceral and subcutaneous abdominal fat was measured using computed tomography. We predicted fibrosis based on the fibrosis-4 (FIB-4) score and aspartate aminotransferase-to-platelet ratio index (APRI) and categorized the risk for advanced fibrosis as low, indeterminate, or high.

Results: The multivariable-adjusted prevalence ratios for indeterminate to high risk of advanced fibrosis based on FIB-4, determined by comparing the second, third, and fourth quartiles with the first quartile of VSR, were 3.38 ( $95 \%$ confidence interval [CI], 0.64 to 17.97), 9.41 (95\% CI, 1.97 to 45.01 ), and 19.34 (95\% CI, 4.06 to 92.18 ), respectively. The multivariable-adjusted prevalence ratios for intermediate to high degree of fibrosis according to APRI also increased across VSR quartiles (5.04 [95\% CI, 2.65 to 9.59], 7.51 [95\% CI, 3.91 to 14.42], and 19.55 [95\% CI, 9.97 to 38.34], respectively). High VSR was more strongly associated with the prevalence of NAFLD in nonobese subjects than in obese subjects, and the associations between VSR and intermediate to high probability of advanced fibrosis in NAFLD were stronger in obese subjects than in nonobese subjects.

Conclusion: High VSR values predicted increased NAFLD risk and advanced fibrosis risk with NAFLD, and the predictive value of VSR for indeterminate to high risk of advanced fibrosis was higher in obese subjects than in nonobese subjects.

Keywords: Non-alcoholic fatty liver disease; Intra-abdominal fat; Liver cirrhosis; Subcutaneous fat, abdominal

Received: 15 October 2019, Revised: 3 January 2020, Accepted: 29 January 2020

Corresponding authors: Seungho Ryu

Department of Occupational and Environmental Medicine, Kangbuk Samsung Hospital, Sungkyunkwan University School of Medicine, 29 Saemunan-ro, Jongno-gu, Seoul 03181, Korea

Tel: +82-2-2001-5237, Fax: +82-2-757-0436, E-mail: sh703.yoo@samsung.com

Won-Young Lee

Division of Endocrinology and Metabolism, Department of Internal Medicine, Kangbuk Samsung Hospital, Sungkyunkwan University School of Medicine, 29 Saemunan-ro, Jongno-gu, Seoul 03181, Korea

Tel: +82-2-2001-2579, Fax: +82-2-2001-1588, E-mail: drlwy@hanmail.net
Copyright $\odot 2020$ Korean Endocrine Society

This is an Open Access article distributed under the terms of the Creative Commons Attribution Non-Commercial License (https://creativecommons.org/ licenses/by-nc/4.0/) which permits unrestricted non-commercial use, distribution, and reproduction in any medium, provided the original work is properly cited.

*These authors contributed equally to this work. 


\section{INTRODUCTION}

Nonalcoholic fatty liver disease (NAFLD) has become a leading cause of chronic liver disease, cirrhosis, type 2 diabetes mellitus (T2DM), and liver-related mortality $[1,2]$. In addition, the presence of NAFLD is associated with higher morbidity and mortality of cardiovascular disease (CVD) than the absence of NAFLD [3].The liver develops fibrosis as a wound-healing process after various kinds of liver injury [4]. Recently, emerging data have suggested that the degree of fibrosis in the presence of NAFLD is an important prognostic factor for future liver-related outcomes and mortality and is associated with the cardiometabolic risk [5,6].

Obesity is highly correlated not only with the NAFLD prevalence and but also with histologic severity $[7,8]$. It is well known that abdominal obesity, especially high visceral adipose tissue (VAT), plays an important role in the development of metabolic diseases and NAFLD independent of generalized obesity [7,9]. Previous studies have demonstrated that VAT is clearly associated with NAFLD. On the other hand, the role of subcutaneous adipose tissue (SAT) in NAFLD is controversial, in that some studies suggested no relationship between SAT and NAFLD [10], and others reported an inverse relationship [1114]. Considering the independent effects of both VAT and SAT on the cardiometabolic risk, the relative distribution of body fat might be more important than VAT or SAT per se. Consequently, the visceral-to-subcutaneous fat ratio (VSR) has been proposed to be a better predictor of atherosclerosis and cardiometabolic risk not only in the asymptomatic general population and obese subjects but also in patients with T2DM [15-18]. However, there are few available studies about the contribution of VSR to NAFLD and liver fibrosis in the general population [14].

Further, although NAFLD is highly associated with obesity, it is also known to be prevalent in nonobese subjects [19]. A metaanalysis study demonstrated that lean and obese subjects with NAFLD share a common altered metabolic and cardiovascular profile [8]. Relative to lean individuals without NAFLD, the lean population with NAFLD showed an excess of abdominal adipose tissue [20]. In particular, studies showing whether body fat distribution, represented by VSR, has a diagnostic value in identifying multiple metabolic risk factors in nonobese subjects, compared with visceral fat area (VFA) and subcutaneous fat area (SFA), are rare. A previous study revealed that VSR showed superior diagnostic value in predicting multiple risk factors in subjects with normal waist circumference and proved that it was superior to VFA [15].
To our knowledge, few studies have examined whether the quartiles of VSR assessed using fat-computed tomography (CT) are associated with advanced fibrosis in the general population with NAFLD. We hypothesized that body fat distribution represented by VSR would indicate the risk for the presence of NAFLD and for advanced fibrosis in individuals with NAFLD. Therefore, we investigated the association of VSR quartiles measured using fat-CT with NAFLD and the probability of advanced fibrosis in the presence of NAFLD by using the fibrosis (FIB-4) score and aspartate aminotransferase (AST)-to-platelet (PLT) ratio index (APRI) in healthy Korean adults. In addition, we further analyzed this association according to the presence or absence of obesity.

\section{METHODS}

\section{Study design and study participants}

In this cross-sectional study, we recruited participants from the Kangbuk Samsung Health Study, a program of the Health Promotion Center of Kangbuk Samsung Hospital, Sungkyunkwan University School of Medicine, Seoul, Korea. The purpose of the medical health checkup program is to promote the health of employees through regular health examinations and to enable the early detection of diseases. Most of the examinees are employees of various industrial companies throughout the country, and their family members. The medical examinations are largely paid for by the employers, and a considerable proportion of the examinees undergo examinations annually or biennially.

The present analysis included participants who underwent fatCT during the health checkup program between January 2013 and December $2017(n=10,229)$. Of these 10,229 subjects, we excluded those with alcohol intake of $\geq 30 \mathrm{~g} /$ day for men or $\geq$ $20 \mathrm{~g} /$ day for women $(n=1,574)$; use of steatogenic medications within the past year, such as valproate, amiodarone, methotrexate, tamoxifen, or corticosteroids $(n=30)$; known liver disease or current use of medications for liver disease $(n=297)$; positive serologic markers for hepatitis $\mathrm{B}$ virus or hepatitis $\mathrm{C}$ virus ( $n=288)$; history of liver cirrhosis or findings of liver cirrhosis on ultrasonography $(n=2)$; and missing data on alcohol status, abdominal ultrasonography, body mass index (BMI), high-sensitivity C-reactive protein (hs-CRP), or homeostasis model assessment of insulin resistance (HOMA-IR) $(n=2,771)$. Final analyses were performed in 7,465 subjects.

This study was approved by the Institutional Review Board of Kangbuk Samsung Hospital (KBSMC 2018-11-003), which waived the requirement for informed consent because we only 
retrospectively accessed a de-identified database for analysis purposes.

\section{Diagnosis of NAFLD and measurement of abdominal fat area}

All subjects underwent abdominal ultrasonography (Logic Q700 MR, GE, Milwaukee, WI, USA). Fatty liver was diagnosed according to standard criteria, including hepatorenal echo contrast, liver brightness, and vascular blurring on ultrasonography with a $3.5-\mathrm{MHz}$ probe, which was conducted by experienced radiologists blinded to the aim of this study and the clinical status of the subjects [21]. NAFLD was defined as the detection of fatty liver in the absence of other etiologies of hepatic steatosis, as described in the exclusion criteria. Of the final 7,465 subjects, 2,706 were diagnosed with NAFLD on ultrasonography.

Abdominal VFA and SFA were measured by CT (a Lightspeed VCT XTe-64 slice Multi-detector CT scanner, GE Healthcare, Tokyo, Japan) [22]. A cross-sectional scan at $10 \mathrm{~mm}$ thickness centered at the L4-L5 vertebral disc space, with the subject in the supine position, was obtained using a radiograph of the skeleton as a reference to establish the position of the scans to the nearest millimeter. SFA and VFA boundaries were defined using a manual cursor by delineating these areas with a graph pen. The adipose tissue areas were calculated by computing the fat area surfaces with an attenuation range of -190 to -30 Hounsfield units. The abdominal VFA was measured by drawing a line in the muscle wall surrounding the abdominal cavity. The SFA was calculated by subtracting the VFA from the total fat area. Then the VSR was calculated.

\section{Estimating the risk of advanced fibrosis in NAFLD}

In subjects with NAFLD, FIB-4, and APRI as serum markers of fibrosis were used to estimate the degree of advanced fibrosis [23]. The FIB-4 score includes readily available clinical data of age, AST, alanine aminotransferase (ALT), and PLT, and was validated for use in NAFLD by Shah et al. [24]. The FIB-4 score was calculated using the following formula: FIB-4=[age $(\mathrm{yr}) \times \operatorname{AST}(\mathrm{IU} / \mathrm{L})] /\left\{\left[\operatorname{PLT}\left(10^{9} / \mathrm{L}\right)\right] \times\left[\operatorname{ALT}(\mathrm{IU} / \mathrm{L})^{2}\right]\right\}$. The cutoffs for low, intermediate, and high probability of advanced fibrosis were $<1.3,1.3$ to 2.67 , and $>2.67$, respectively. The APRI is another simple index for identifying patients with advanced fibrosis ( $\geq$ F3) and was calculated using the following formula: APRI $=\left[\right.$ AST $/$ AST $($ upper limit of normal) $] /$ PLT $\left(\times 10^{9} / \mathrm{L}\right)$ $[25,26]$. We used the cutoff of 0.5 for low probability and 1.5 for high probability of advanced fibrosis.

\section{Covariates and baseline anthropometric and laboratory measurements}

The values of serum biochemical parameters and physical measurements were obtained by trained staff during health examinations, and data on medical history, medication use, and healthrelated behaviors were collected through a self-administered questionnaire. BMI was calculated by dividing weight $(\mathrm{kg})$ by the square of height $\left(\mathrm{m}^{2}\right)$. Systolic blood pressure and diastolic blood pressure were measured three times with the subjects in a seated position and with a 1-minute rest between each measurement. The average of the second and third measurements was used in our analyses.

Blood samples were collected after 12 hours of overnight fasting. Fasting plasma glucose levels were determined through the hexokinase method (Modular D2400, Hitachi, Tokyo, Japan). An electrochemiluminescence immunoassay was used to assess fasting insulin levels (Modular E170, Hitachi). An enzymatic colorimetric test was used to measure the total cholesterol and triglyceride levels. The selective inhibition method was used to measure high density lipoprotein cholesterol levels, and a homogeneous enzymatic colorimetric test was used to measure low density lipoprotein cholesterol levels. ALT, AST, and gamma-glutamyltransferase were measured using Bayer Reagent Packs on an automated chemistry analyzer (Advia 1650 autoanalyzer, Bayer Diagnostics, Leverkusen, Germany). Serum hs-CRP levels were measured using a nephelometric assay with a BNII nephelometer (Dade Behring, Deerfield, IL, USA). Insulin resistance was measured using the HOMA-IR and was obtained by applying the following formula: HOMA-IR = fasting insulin $(\mu \mathrm{IU} / \mathrm{mL}) \times$ fasting blood glucose $(\mathrm{mmol} / \mathrm{L}) / 22.5$ [27].

A smoker was defined as a subject who had smoked at least five packs of cigarettes over the course of the lifetime. Regular exercise was defined as moderate-intensity exercise at least three times every week.

Total energy and nutrient intake was calculated by using food composition table developed by the Korean Nutrition Society [28]. Education level and history of CVD were evaluated using self-reported questionnaire.

Hypertension was defined as systolic blood pressure $\geq 140$ $\mathrm{mm} \mathrm{Hg}$, diastolic blood pressure $\geq 90 \mathrm{~mm} \mathrm{Hg}$, self-reported history of hypertension, or current use of antihypertensive medications. The presence of diabetes was defined as fasting plasma glucose level $\geq 126 \mathrm{mg} / \mathrm{dL}$ or hemoglobin A1c level $\geq 6.5 \%$, a self-reported history of diabetes, or current use of antidiabetic medication [29]. 


\section{Statistical analysis}

The characteristics of the study participants were examined according to VSR quartiles. To test for linear trends, category numbers were used as continuous variables in regression models. The study population was grouped into quartiles of VSR, as follows: Q1 (<0.37), Q2 (0.37 to 0.51), Q3 (0.52 to 0.70$)$, and Q4 ( $\geq 0.71)$.

To evaluate the association of NAFLD across quartiles of VSR, SFA, and VFA, we used Poisson regression with robust variance to estimate prevalence ratios (PRs) with 95\% confidence intervals (CIs) for NAFLD. Models were initially adjusted for age and sex, and further adjusted for center (Seoul or Suwon), year of screening examination, smoking status (never, past, current, or unknown), physical activity, alcohol intake ( 0 , $<10,>10 \mathrm{~g} /$ day, or unknown), total energy intake (in quintile or unknown), education level, history of CVD, history of diabetes, and history of hypertension (model 1). To investigate whether this association was influenced by obesity or insulin resistance, we performed additional analysis with adjustment for BMI and HOMA-IR in model 2. We also estimated the PRs of NAFLD with the degree of fibrosis based on FIB-4 or APRI by VSR quartiles, by using multinomial logistic regression models as outcomes categorized into no NAFLD, NAFLD plus low FIB-4 or APRI, and NAFLD plus intermediate/high FIB-4 or APRI. Multivariable models 1 and 2 were adjusted with the same variables described above. To determine the linear trends of prevalence, the number of quartiles was used as a continuous variable and tested on each model.

We performed stratified analyses in prespecified subgroups defined by BMI ( $<25 \mathrm{~kg} / \mathrm{m}^{2}$ vs. $\left.\geq 25 \mathrm{~kg} / \mathrm{m}^{2}\right)$. Interactions between subgroups were tested using likelihood ratio tests comparing models with and without multiplicative interaction terms. All $P$ values were two-tailed, and values of $P<0.05$ were considered statistically significant. We used STATA version 14.0 (Stata Corp., College Station, TX, USA) for data analysis.

\section{RESULTS}

\section{General characteristics of the participants according to VSR quartiles}

The total number of subjects was 7,465 (5,532 men and 1,933 women), and their mean age was 35.7 years (Table 1). The subjects were subdivided into four groups according to VSR quartiles. The baseline characteristics according to VSR quartiles are presented in Table 1.

The proportion of men, subjects with a history of diabetes, subjects with hypertension, and subjects with CVD significantly increased across VSR quartiles. BMI, insulin resistance, and the mean values of glucose and traditional and nontraditional cardiovascular risk factors (blood pressure, lipid profile, and hsCRP) significantly increased across VSR quartiles. The AST level, ALT level, FIB-4, and APRI significantly increased across VSR quartile groups.

\section{Risk of incident NAFLD across quartiles of VSR, SAT, and VAT}

Of the total 7,465 subjects, 2,706 (36.2\%) were diagnosed with NAFLD on ultrasonography. Table 2 presents the risk of incident NAFLD across the quartiles of VSR. In age- and sex-adjusted models, the PRs for NAFLD comparing Q2 to Q4 withQ1 of VSR were 1.4 (95\% CI, 1.23 to 1.59$), 1.67$ (95\% CI, 1.47 to 1.89$)$, and 1.75 (95\% CI, 1.54 to 1.99$)$, respectively. After further adjusting for confounding variables including examination center, year of screening examination, smoking status, physical activity, alcohol intake, education level, history of CVD, history of diabetes, and history of hypertension, significantly increased PRs were sustained across the groups. The results were strengthened after additional adjustment for BMI and HOMA-IR (1.54 vs. 1.95 vs. 2.24). When the analyses were performed according to gender after excluding 151 subjects with diabetes, women showed higher PRs in all analyses compared with men, although the observed trends were similar (Supplemental Table S1).

The PRs of NAFLD were analyzed according to quartiles of SAT and VAT (Table 2). The prevalence of NAFLD also significantly increased across the quartiles of subcutaneous and visceral fat.

\section{Prevalence of NAFLD and degree of fibrosis based on fibrosis scores according to VSR quartiles}

We assessed the degree of advanced fibrosis in subjects with NAFLD based on FIB-4 and APRI, and categorized the subjects into having a low, intermediate, or high probability of fibrosis. Table 3 shows the association between VSR quartiles and the degree of advanced fibrosis assessed by FIB-4 in NAFLD by using multinomial logistic regression. The age- and sex-adjusted PRs $(95 \%$ CI) for NAFLD with low FIB-4 comparing Q2 to Q4 with Q1 of VSR increased steadily (1.51 [95\% CI, 1.27 to 1.8], 2.0 [95\% CI, 1.67 to 2.38], and 2.19 [95\% CI, 1.81 to $2.64]$, respectively). This association also persisted even after further adjustment for the above-mentioned confounders in model 1 and for BMI and HOMA-IR (2.23 [95\% CI, 1.8 to 
Table 1. Baseline Characteristics by VSR Quartiles

\begin{tabular}{|c|c|c|c|c|c|c|}
\hline \multirow{2}{*}{ Characteristic } & \multirow{2}{*}{ Overall } & \multicolumn{4}{|c|}{ VSR quartile } & \multirow{2}{*}{$\begin{array}{l}P \text { for } \\
\text { trend }\end{array}$} \\
\hline & & Q1 $(<0.37)$ & Q2 (0.37-0.51) & Q3 (0.52-0.70) & $\mathrm{Q} 4(\geq 0.71)$ & \\
\hline No. of participants & 7,465 & 1,953 & 1,832 & 1,848 & 1,832 & \\
\hline Age, yr & $35.7 \pm 7.2$ & $32.4 \pm 5.7$ & $34.6 \pm 6.3$ & $36.3 \pm 7.0$ & $39.8 \pm 7.7$ & $<0.001$ \\
\hline Seoul center & 36.7 & 31.0 & 31.5 & 36.7 & 47.8 & $<0.001$ \\
\hline Men, $\%$ & 74.1 & 37.5 & 72.2 & 91.6 & 97.3 & $<0.001$ \\
\hline Alcohol intake,$\%$ & 35.4 & 25.0 & 34.0 & 40.5 & 42.5 & $<0.001$ \\
\hline Current smoker, \% & 17.5 & 9.0 & 16.1 & 21.5 & 23.7 & $<0.001$ \\
\hline Education level,$\%$ & 90.3 & 84.8 & 90.5 & 92.2 & 93.9 & $<0.001$ \\
\hline Diabetes, $\%$ & 2.1 & 0.4 & 1.3 & 2.5 & 4.3 & $<0.001$ \\
\hline Hypertension, \% & 9.1 & 4.5 & 6.4 & 10.9 & 14.7 & $<0.001$ \\
\hline SBP, mm Hg & $110.1 \pm 11.3$ & $105.7 \pm 11.5$ & $109.5 \pm 10.6$ & $112.7 \pm 10.9$ & $113.0 \pm 10.4$ & $<0.001$ \\
\hline DBP, mm Hg & $69.9 \pm 8.9$ & $66.1 \pm 8.2$ & $68.5 \pm 8.3$ & $71.6 \pm 8.7$ & $73.5 \pm 7.5$ & $<0.001$ \\
\hline FPG, mg/dL & $92.6 \pm 11.7$ & $89.7 \pm 7.5$ & $92.0 \pm 9.8$ & $93.8 \pm 13.9$ & $95.0 \pm 13.9$ & $<0.001$ \\
\hline $\mathrm{TC}, \mathrm{mg} / \mathrm{dL}$ & $196.1 \pm 35.0$ & $187.7 \pm 32.8$ & $194.7 \pm 33.8$ & $199.9 \pm 35.7$ & $202.7 \pm 35.8$ & $<0.001$ \\
\hline LDL-C, mg/dL & $128.6 \pm 33.4$ & $115.9 \pm 30.5$ & $127.8 \pm 32.0$ & $134.2 \pm 33.6$ & $137.3 \pm 33.5$ & $<0.001$ \\
\hline HDL-C, mg/dL & $57.3 \pm 15.2$ & $65.8 \pm 16.2$ & $57.4 \pm 14.3$ & $53.6 \pm 12.8$ & $51.7 \pm 12.9$ & $<0.001$ \\
\hline Triglycerides, mg/dL & $97(68-144)$ & $71(55-97)$ & $94(68-133)$ & $112(80-160)$ & $128(89-177)$ & $<0.001$ \\
\hline AST, U/L & $20(17-26)$ & $18(16-22)$ & $20(17-25)$ & $21(18-27)$ & $22(18-28)$ & $<0.001$ \\
\hline ALT, U/L & $21(15-33)$ & $15(11-23)$ & $21(15-31)$ & $24(17-37)$ & $26(19-40)$ & $<0.001$ \\
\hline GGT, U/L & $23(15-37)$ & $15(12-22)$ & $21(15-33)$ & $26(18-42)$ & $31(21-47)$ & $<0.001$ \\
\hline hs-CRP, mg/L & $0.5(0.3-0.9)$ & $0.3(0.2-0.7)$ & $0.4(0.3-0.9)$ & $0.5(0.3-1.0)$ & $0.6(0.3-1.1)$ & $<0.001$ \\
\hline HOMA-IR & $1.30(0.89-1.90)$ & $1.18(0.79-1.72)$ & $1.30(0.89-1.89)$ & $1.39(0.94-2.02)$ & $1.35(0.94-1.95)$ & $<0.001$ \\
\hline Platelets, $\times 10^{3} / \mathrm{mm}^{3}$ & $250(220-285)$ & $254(221-289)$ & $248(220-285)$ & $250(219-282)$ & $248(218-285)$ & 0.003 \\
\hline $\begin{array}{l}\text { Total energy intake, } \\
{\mathrm{kcal} / \text { day }^{\mathrm{c}}}\end{array}$ & $1,359.3(1,030.9-1,758.8)$ & $1,242.8(895.9-1,652.7)$ & $1,383.4(1,031.8-1,784.7)$ & $1,435.4(1,125.8-1,828.7)$ & $1,384.9(1,091.3-1,776.0)$ & $<0.001$ \\
\hline FIB-4 & $0.68 \pm 0.28$ & $0.63 \pm 0.24$ & $0.66 \pm 0.25$ & $0.68 \pm 0.29$ & $0.75 \pm 0.30$ & $<0.001$ \\
\hline APRI & $0.25 \pm 0.17$ & $0.24 \pm 0.12$ & $0.25 \pm 0.18$ & $0.26 \pm 0.22$ & $0.26 \pm 0.15$ & $<0.001$ \\
\hline
\end{tabular}

Values are expressed as mean \pm standard deviation, percentage, or median (interquartile range).

VSR, visceral-to-subcutaneous fat ratio; SBP, systolic blood pressure; DBP, diastolic blood pressure; FPG, fasting plasma glucose; TC, total cholesterol; LDL-C, low density lipoprotein cholesterol; HDL-C, high density lipoprotein cholesterol; AST, aspartate aminotransferase; ALT, alanine aminotransferase; GGT, gamma-glutamyltransferase; hs-CRP, high-sensitivity C-reactive protein; HOMA-IR, homeostasis model assessment of insulin resistance; FIB-4, fibrosis-4 score; APRI, aspartate aminotransferase-to-platelet ratio index; SD, standard deviation.

${ }^{\mathrm{a}} \geq 10 \mathrm{~g}$ of ethanol per day; ${ }^{\mathrm{b}} \mathrm{College}$ graduate or higher; ${ }^{\mathrm{C}}$ Among 5,523 participants with plausible estimated energy intake (within $3 \mathrm{SDs}$ of the log-transformed mean energy intake).

2.76], 3.8 [95\% CI, 3.04 to 4.73$]$, and 5.26 [95\% CI, 4.15 to 6.68], respectively). With respect to the PRs for NAFLD with intermediate or high FIB-4, the age- and sex-adjusted PRs substantially increased across VSR quartiles $(1.7[95 \% \mathrm{CI}, 0.34$ to 8.59] for Q2, 3.48 [95\% CI, 0.76 to 15.89$]$ for Q3, and 5.79 [95\% CI, 1.28 to 26.14] for Q4, respectively). The results were strengthened after multivariable adjustment including model 1 plus BMI and HOMA-IR (19.34 [95\% CI, 4.06 to 92.18] for Q4; $P$ for trend $<0.001)$.
Table 4 shows the association between VSR quartiles and the degree of advanced fibrosis based on APRI in subjects with NAFLD. The results were similar to those based on FIB-4. The PRs $(95 \% \mathrm{CI})$ for NAFLD with low APRI comparing Q2, Q3, and Q4 with Q1 of VSR moderately increased after multivariate covariate adjustment (1.45 [95\% CI, 1.21 to 1.73$], 1.96$ [95\% CI, 1.64 to 2.35], and 2.1 [95\% CI, 1.73 to 2.55 ], respectively). This association persisted even after further adjustment for BMI and HOMA-IR (2.14 [95\% CI, 1.72 to 2.65], 3.67 [95\% CI, 2.94 
Table 2. Prevalence Ratios ${ }^{\mathrm{a}}(95 \%$ CI) of Nonalcoholic Fatty Liver Disease by Quartiles of VSR, Subcutaneous Fat, and Visceral Fat

\begin{tabular}{|c|c|c|c|c|c|}
\hline \multirow{2}{*}{ Variable } & \multirow{2}{*}{ Number } & \multirow{2}{*}{ Case } & \multirow{2}{*}{$\begin{array}{l}\text { Age/sex-adjusted } \mathrm{PR}^{\mathrm{a}} \\
(95 \% \mathrm{CI})\end{array}$} & \multicolumn{2}{|c|}{ Multivariate-adjusted $\mathrm{PR}^{\mathrm{a}}$} \\
\hline & & & & Model 1 & Model 2 \\
\hline \multicolumn{6}{|l|}{ VSR quartiles } \\
\hline Q1 (<0.37) & 1,953 & 295 & 1.00 (reference) & 1.00 (reference) & 1.00 (reference) \\
\hline Q2 (0.37-0.51) & 1,832 & 575 & $1.40(1.23-1.59)$ & $1.38(1.22-1.56)$ & $1.54(1.37-1.74)$ \\
\hline Q3 (0.52-0.70) & 1,848 & 844 & $1.67(1.47-1.89)$ & $1.61(1.42-1.82)$ & $1.95(1.75-2.19)$ \\
\hline $\mathrm{Q} 4(\geq 0.71)$ & 1,832 & 992 & $1.75(1.54-1.99)$ & $1.67(1.47-1.90)$ & $2.24(1.99-2.52)$ \\
\hline$P$ for trend & & & $<0.001$ & $<0.001$ & $<0.001$ \\
\hline \multicolumn{6}{|c|}{ Subcutaneous fat quartiles } \\
\hline Q1 $(<6,058)$ & 1,867 & 256 & 1.00 (reference) & 1.00 (reference) & 1.00 (reference) \\
\hline Q2 $(6,058-9,142)$ & 1,866 & 535 & $1.94(1.71-2.21)$ & $1.91(1.69-2.17)$ & $1.60(1.41-1.82)$ \\
\hline Q3 $(9,143-12,597)$ & 1,866 & 801 & $2.83(2.52-3.19)$ & $2.73(2.42-3.07)$ & $1.96(1.73-2.22)$ \\
\hline Q4 ( $\geq 12,598)$ & 1,866 & 1,114 & $4.03(3.59-4.51)$ & $3.83(3.42-4.30)$ & $1.90(1.65-2.18)$ \\
\hline$P$ for trend & & & $<0.001$ & $<0.001$ & $<0.001$ \\
\hline \multicolumn{6}{|l|}{ Visceral fat quartiles } \\
\hline Q1 $(<13,589)$ & 1,868 & 48 & 1.00 (reference) & 1.00 (reference) & 1.00 (reference) \\
\hline Q2 (13,589-17,302) & 1,865 & 383 & $7.20(5.32-9.75)$ & $7.14(5.27-9.66)$ & $5.73(4.24-7.73)$ \\
\hline Q3 (17,303-21,834) & 1,866 & 869 & $15.60(11.54-21.10)$ & $15.31(11.33-20.70)$ & $10.63(7.87-14.36)$ \\
\hline Q4 ( $\geq 21,835)$ & 1,866 & 1,406 & $24.85(18.37-33.61)$ & $24.22(17.90-32.76)$ & $13.74(10.12-18.65)$ \\
\hline$P$ for trend & & & $<0.001$ & $<0.001$ & $<0.001$ \\
\hline
\end{tabular}

VSR, visceral-to-subcutaneous fat ratio; CI, confidence interval; PR, prevalence ratio.

${ }^{a}$ Estimated from Poisson regression with robust error. Multivariable model 1: age, sex, center, year of screening examination, smoking status, physical activity, alcohol intake, education level, history of cardiovascular disease, history of diabetes, and history of hypertension; model 2: model 1 plus adjustment for body mass index and homeostasis model assessment of insulin resistance.

to 4.58 ], and 4.99 [95\% CI, 3.93 to 6.33 ], respectively). In addition, the risk for NAFLD with intermediate or high APRI significantly increased across VSR quartiles and was strengthened after further adjustments for BMI and HOMA-IR (5.04 [95\% CI, 2.65 to 9.59], 7.51 [95\% CI, 3.91 to 14.42 ], and 19.55 [95\% CI, 9.97 to 38.34$]$, respectively).

\section{Prevalence of NAFLD and degree of fibrosis by VSR quartiles according to obesity}

The association of VSR quartiles with NAFLD and the risk for advanced fibrosis was also examined by subgroups of study participants according to obesity (Table 5). These associations of VSR quartiles with NAFLD and the intermediate to high probability of advanced fibrosis in NAFLD were modified by obesity ( $P$ for interaction $<0.01$ ). High VSR values were more strongly associated with the prevalence of NAFLD in nonobese than in obese subjects. In addition, the associations between VSR values and the intermediate to high probability of advanced fibrosis assessed by FIB-4 in NAFLD were stronger in obese than in nonobese subjects. With respect to the PRs for advanced fibrosis based on APRI, the results were similar to those for FIB-4.

\section{DISCUSSION}

The results of the present cross-sectional study show that high VSR is an independent risk factor for the presence of NAFLD and advanced fibrosis even after adjustment for BMI and insulin resistance. In a subgroup analysis according to obesity, VSR was well associated with NAFLD even in nonobese subjects and the PR for NAFLD was higher in the nonobese than in the obese group. Concerning the probability of advanced fibrosis, higher VSR was more strongly associated with an intermediate to high probability of advanced fibrosis, especially in the obese group.

Previous epidemiologic, cross-sectional, and longitudinal studies demonstrated that VAT is clearly associated with NAFLD, whereas the role of SAT in NAFLD remains inconclu- 
Table 3. Prevalence Ratios ${ }^{\mathrm{a}}$ (95\% CI) of NAFLD and Degree of Fibrosis Based on FIB-4 Score by VSR Quartiles

\begin{tabular}{|c|c|c|c|c|c|}
\hline \multirow{2}{*}{ VSR quartile } & \multirow{2}{*}{ Number } & \multirow{2}{*}{ Case } & \multirow{2}{*}{$\begin{array}{l}\text { Age/sex-adjusted } \mathrm{PR}^{\mathrm{a}} \\
\qquad(95 \% \mathrm{CI})\end{array}$} & \multicolumn{2}{|c|}{ Multivariate-adjusted $\mathrm{PR}^{\mathrm{a}}$} \\
\hline & & & & Model 1 & Model 2 \\
\hline \multicolumn{6}{|c|}{ NAFLD+low FIB-4 } \\
\hline Q1 $(<0.37)$ & 1,953 & 293 & 1.00 (reference) & 1.00 (reference) & 1.00 (reference) \\
\hline Q2 (0.37-0.51) & 1,832 & 569 & $1.52(1.28-1.81)$ & $1.51(1.27-1.80)$ & $2.23(1.80-2.76)$ \\
\hline Q3 (0.52-0.70) & 1,848 & 826 & $2.07(1.74-2.45)$ & $2.00(1.67-2.38)$ & $3.80(3.04-4.73)$ \\
\hline $\mathrm{Q} 4(\geq 0.71)$ & 1,832 & 943 & $2.31(1.92-2.77)$ & $2.19(1.81-2.64)$ & $5.26(4.15-6.68)$ \\
\hline$P$ for trend & & & $<0.001$ & $<0.001$ & $<0.001$ \\
\hline \multicolumn{6}{|c|}{ NAFLD+intermediate/high FIB-4 } \\
\hline Q1 $(<0.37)$ & 1,953 & 2 & 1.00 (reference) & 1.00 (reference) & 1.00 (reference) \\
\hline Q2 (0.37-0.51) & 1,832 & 6 & $1.70(0.34-8.59)$ & $2.05(0.40-10.67)$ & $3.38(0.64-17.97)$ \\
\hline Q3 (0.52-0.70) & 1,848 & 18 & $3.48(0.76-15.89)$ & $4.45(0.93-21.20)$ & $9.41(1.97-45.01)$ \\
\hline $\mathrm{Q} 4(\geq 0.71)$ & 1,832 & 48 & $5.79(1.28-26.14)$ & $7.40(1.56-35.06)$ & $19.34(4.06-92.18)$ \\
\hline$P$ for trend & & & $<0.001$ & $<0.001$ & $<0.001$ \\
\hline
\end{tabular}

CI, confidence interval; NAFLD, nonalcoholic fatty liver disease; FIB-4, fibrosis-4; VSR, visceral-to-subcutaneous fat ratio; PR, prevalence ratio. ${ }^{a}$ Estimated from multinomial logistic regression models as outcomes categorized as no NAFLD, NAFLD plus low FIB-4, and NAFLD plus intermediate/high FIB-4. Multivariable model 1 was adjusted for age, sex, center, year of screening examination, smoking status, physical activity, alcohol intake, total energy intake, education level, history of cardiovascular disease, history of diabetes, and history of hypertension; model 2: model 1 plus adjustment for body mass index and homeostasis model assessment of insulin resistance.

Table 4. Prevalence Ratios ${ }^{\mathrm{a}}(95 \%$ CI) of NAFLD and Degree of Fibrosis Based on APRI by VSR Quartiles

\begin{tabular}{|c|c|c|c|c|c|}
\hline \multirow{2}{*}{ VSR quartile } & \multirow{2}{*}{ Number } & \multirow{2}{*}{ Case } & \multirow{2}{*}{$\begin{array}{c}\text { Age/sex-adjusted } \mathrm{PR}^{\mathrm{a}} \\
(95 \% \mathrm{CI})\end{array}$} & \multicolumn{2}{|c|}{ Multivariate-adjusted $\mathrm{PR}^{\mathrm{a}}$} \\
\hline & & & & Model 1 & Model 2 \\
\hline \multicolumn{6}{|c|}{ NAFLD+low APRI } \\
\hline Q1 $(<0.37)$ & 1,953 & 279 & 1.00 (reference) & 1.00 (reference) & 1.00 (reference) \\
\hline Q2 (0.37-0.51) & 1,832 & 526 & $1.46(1.23-1.74)$ & $1.45(1.21-1.73)$ & $2.14(1.72-2.65)$ \\
\hline Q3 (0.52-0.70) & 1,848 & 789 & $2.03(1.70-2.42)$ & $1.96(1.64-2.35)$ & $3.67(2.94-4.58)$ \\
\hline $\mathrm{Q} 4(\geq 0.71)$ & 1,832 & 903 & $2.22(1.85-2.68)$ & $2.10(1.73-2.55)$ & $4.99(3.93-6.33)$ \\
\hline$P$ for trend & & & $<0.001$ & $<0.001$ & $<0.001$ \\
\hline \multicolumn{6}{|c|}{ NAFLD+intermediate/high APRI } \\
\hline $\mathrm{Q} 1(<0.37)$ & 1,953 & 16 & 1.00 (reference) & 1.00 (reference) & 1.00 (reference) \\
\hline Q2 (0.37-0.51) & 1,832 & 49 & $2.46(1.37-4.42)$ & $2.56(1.42-4.63)$ & $5.04(2.65-9.59)$ \\
\hline Q3 $(0.52-0.70)$ & 1,848 & 55 & $2.67(1.47-4.84)$ & $2.70(1.48-4.93)$ & $7.51(3.91-14.42)$ \\
\hline $\mathrm{Q} 4(\geq 0.71)$ & 1,832 & 89 & $4.53(2.50-8.21)$ & $4.71(2.56-8.66)$ & $19.55(9.97-38.34)$ \\
\hline$P$ for trend & & & $<0.001$ & $<0.001$ & $<0.001$ \\
\hline
\end{tabular}

CI, confidence interval; NAFLD, nonalcoholic fatty liver disease; APRI, aspartate aminotransferase-to-platelet ratio index; VSR, visceral-to-subcutaneous fat ratio; PR, prevalence ratio.

aEstimated from multinomial logistic regression models as outcomes categorized as no NAFLD, NAFLD plus low APRI, and NAFLD plus intermediate/ high APRI. Multivariable model 1 was adjusted for age, sex, center, year of screening examination, smoking status, physical activity, alcohol intake, total energy intake, education level, history of cardiovascular disease, history of diabetes, and history of hypertension; model 2: model 1 plus adjustment for body mass index and homeostasis model assessment of insulin resistance.

sive [11]. Some studies showed that SAT is correlated with NAFLD, whereas others did not find this correlation [12]. How- ever, there are few studies about the association of VSR and NAFLD and the degree of advanced fibrosis [12,14]. In a study 
Table 5. Prevalence Ratios ${ }^{\mathrm{a}}(95 \%$ CI) of NAFLD or NAFLD and Degree of Fibrosis by VSR Quartiles According to Obesity

\begin{tabular}{|c|c|c|c|}
\hline \multirow{2}{*}{ VSR quartile } & \multicolumn{2}{|c|}{ Multivariate-adjusted PR } & \multirow{2}{*}{$P$ for interaction } \\
\hline & Nonobese $(n=4,679)$ & Obese $(n=2,786)$ & \\
\hline \multicolumn{4}{|l|}{ NAFLD } \\
\hline Q1 $(<0.37)$ & 1.00 (reference) & 1.00 (reference) & $<0.001$ \\
\hline Q2 (0.37-0.51) & $2.93(2.15-4.00)$ & $1.23(1.10-1.38)$ & \\
\hline Q3 $(0.52-0.70)$ & $4.57(3.37-6.21)$ & $1.43(1.29-1.59)$ & \\
\hline $\mathrm{Q} 4(\geq 0.71)$ & $6.52(4.81-8.82)$ & $1.38(1.24-1.53)$ & \\
\hline$P$ for trend & $<0.001$ & $<0.001$ & \\
\hline \multicolumn{4}{|c|}{ NAFLD and degree of fibrosis based on FIB-4 } \\
\hline NAFLD+low FIB-4 & & & $<0.001$ \\
\hline Q1 $(<0.37)$ & 1.00 (reference) & 1.00 (reference) & \\
\hline Q2 (0.37-0.51) & $2.81(2.00-3.95)$ & - & \\
\hline Q3 $(0.52-0.70)$ & $4.56(3.26-6.40)$ & - & \\
\hline $\mathrm{Q} 4(\geq 0.71)$ & $7.16(5.09-1.07)$ & - & \\
\hline$P$ for trend & $<0.001$ & $<0.001$ & \\
\hline \multicolumn{4}{|c|}{ NAFLD+intermediate/high FIB-4 } \\
\hline Q1 $(<0.37)$ & 1.00 (reference) & 1.00 (reference) & \\
\hline Q2 (0.37-0.51) & $1.61(1.27-2.03)$ & $2.90(0.53-15.92)$ & \\
\hline Q3 $(0.52-0.70)$ & $2.78(2.18-3.56)$ & $7.33(1.48-36.28)$ & \\
\hline $\mathrm{Q} 4(\geq 0.71)$ & $2.70(2.08-3.51)$ & $8.79(1.80-43.06)$ & \\
\hline$P$ for trend & 0.003 & $<0.001$ & \\
\hline \multicolumn{4}{|c|}{ NAFLD and degree of fibrosis based on APRI } \\
\hline NAFLD+low APRI & & & $<0.001$ \\
\hline Q1 $(<0.37)$ & 1.00 (reference) & 1.00 (reference) & \\
\hline Q2 (0.37-0.51) & $2.74(1.95-3.85)$ & - & \\
\hline Q3 (0.52-0.70) & $4.38(3.13-6.15)$ & - & \\
\hline $\mathrm{Q} 4(\geq 0.71)$ & $6.75(4.80-9.50)$ & - & \\
\hline$P$ for trend & $<0.001$ & $<0.001$ & \\
\hline \multicolumn{4}{|c|}{ NAFLD+intermediate/high APRI } \\
\hline $\mathrm{Q} 1(<0.37)$ & 1.00 (reference) & 1.00 (reference) & \\
\hline Q2 (0.37-0.51) & $1.51(1.19-1.92)$ & $3.14(1.72-5.74)$ & \\
\hline Q3 (0.52-0.70) & $2.70(2.10-3.46)$ & $4.47(2.41-8.29)$ & \\
\hline $\mathrm{Q} 4(\geq 0.71)$ & $2.53(1.94-3.31)$ & $7.01(3.72-13.21)$ & \\
\hline$P$ for trend & $<0.001$ & $<0.001$ & \\
\hline
\end{tabular}

CI, confidence interval; NAFLD, nonalcoholic fatty liver disease; VSR, visceral-to-subcutaneous fat ratio; PR, prevalence ratio; FIB-4, fibrosis-4; APRI, aspartate aminotransferase-to-platelet ratio index.

a'Estimated from Poisson regression with robust error. The multivariable model was adjusted for age, sex, center, year of screening examination, smoking status, physical activity, alcohol intake, total energy intake, education level, history of cardiovascular disease, history of diabetes, and history of hypertension.

of 38 patients with biopsy-proven NAFLD by van der Poorten et al. [12], VSR assessed using magnetic resonance imaging also had an association with increasing fibrosis stage, although the extent of association was less than that of visceral fat. VSR has a moderate negative correlation with adiponectin and leptin but shares similar associations to visceral fat. In addition, subcutaneous fat is not associated with increasing steatosis, inflammation, or fibrosis, indicating its metabolically protective prop- 
erties. Kim et al. [14] investigated the longitudinal association between VAT versus SAT and the incidence and regression of NAFLD in 2017 individuals who participated in a voluntary follow-up health screening with a 4.43-year of median followup. They showed that an increased VAT area was associated with a higher incidence of NAFLD and an increased SAT area was significantly associated with regression of NAFLD in multivariable analysis. Consistent with our study, their study showed that the VSR was strongly associated with incident NAFLD in the fully adjusted multivariate model. However, they did not investigate the association with fibrosis.

Obesity is highly correlated not only with NAFLD prevalence but also with histologic severity. In a systematic review and meta-analysis using cross-sectional data of patients with biopsyproven nonalcoholic steatohepatitis (NASH) and those with no NASH, being overweight or obese was associated with more severe histologic features [8]. Although the mechanisms underlying the association between excessive adiposity or fat gain and the progression of fibrosis are not yet fully understood, insulin resistance and inflammation are believed to be involved [30-32]. However, after adjustment for insulin resistance represented by HOMA-IR, the association between VSR and the risk of advanced fibrosis remained significant in the present study. Multiple other factors, including lipotoxicity, inflammatory attacks by proinflammatory cytokines, adipocytokines, and oxidative stress, may lead to the development of fibrosis in a substantial proportion of the population with NAFLD $[31,33]$.

In this study, higher VSR was significantly and independently associated with a higher risk of NAFLD development even after adjustment for confounders including BMI (as a simple indicator of obesity). In the subgroup analysis according to obesity, the predictive value of VSR for the risk of NAFLD was stronger in the nonobese group than in the obese group. The growing prevalence of NAFLD (around $8 \%$ to $19 \%$ ) in lean Asians with BMI $<25 \mathrm{~kg} / \mathrm{m}^{2}$ has been reported, compared with the prevalence of $-7 \%$ in the United States population $[34,35]$. In a metaanalysis, lean and obese subjects with NAFLD showed similar altered metabolic and cardiovascular profiles [8]. Relative to lean subjects without NAFLD, the lean NAFLD population showed an excess of abdominal adipose tissue. In particular, studies on whether the body fat distribution (represented by VSR) has diagnostic value in identifying multiple metabolic risk factors in nonobese subjects, compared with VFA and SFA, are rare. One previous study revealed that VSR showed superior diagnostic value in predicting multiple risk factors in subjects with normal waist circumference, and proved that it is superior to VFA [15].

Whether nonobese individuals with NAFLD are protected from severe histologic outcomes is unknown. In our study, both nonobese and obese subjects with NAFLD exhibited a high risk of advanced fibrosis across VSR quartiles. Nonetheless, obese NAFLD subjects showed higher odds of having a higher risk for advanced fibrosis according to VSR quartiles than nonobese NAFLD subjects. Some previous studies reported findings similar to our results. A meta-analysis showed that overweight/obese NAFLD subjects based on BMI showed significantly higher fibrosis scores relative to lean subjects (BMI $\leq 25 \mathrm{~kg} / \mathrm{m}^{2}$ ) with NAFLD [8]. Previously, our group evaluated the association of baseline BMI and weight change with fibrosis progression, determined by APRI, in a prospective large cohort study on NAFLD [36]. We found obesity and weight gain to be independently associated with an increased risk of fibrosis progression, from a low to an intermediate or a high probability of advanced fibrosis.

This study has several limitations. First, NAFLD and liver fibrosis were diagnosed using ultrasonography and calculated indices, not with biopsy. Furthermore, we could not consider the severity of NAFLD diagnosed with ultrasonography due to relative imperfectness of ultrasonography to diagnose fatty liver. Although liver biopsy is required for an accurate diagnosis and staging of liver fibrosis, it is not practical to perform liver biopsies in all low-risk individuals, because of several challenges such as sampling errors, intra-observer variations, invasiveness, and risk of complication [37]. Therefore, simple serum-based noninvasive scoring systems such as FIB-4 and APRI have been developed and validated to identify and exclude advanced fibrosis in patients with NAFLD, and have been proven to correlate well with liver histology $[23,38,39]$. In addition, evidence for advanced fibrosis as determined using noninvasive scoring systems and markers is useful in predicting the increased risk of liver-related complications and cardiovascular mortality [40,41]. Second, as this was a cross-sectional study, the causal relationship between VSR and NAFLD or advanced fibrosis in NAFLD cannot be determined. Third, the generalization of our findings may be limited by the study population comprising young and middle-aged healthy Koreans. Lastly, as the information regarding the menopausal status of the female participants were not accurate, we could not analyze the data of pre- and postmenopausal women separately. Although the strength of this study is the large sample size, we could not perform stratified subgroup analyses with the PR of the low risk of fibrosis according to VSR quartiles in the obese group because of the small number 
of obese subjects with a low risk of fibrosis.

In conclusion, we have demonstrated that VSR is associated with NAFLD and advanced fibrosis, based on FIB-4 or APRI, in subjects with NAFLD. This effect was independent of age, sex, insulin resistance, and obesity. In stratified analysis, VSR more strongly predicted the risk of NAFLD in the nonobese group than in the obese group. Additionally, the predictive value of VSR for intermediate to high risk of advanced fibrosis was higher in the obese group than in the nonobese group. Future large prospective studies are required to confirm the predictive value of VSR for NAFLD and advanced fibrosis in NAFLD in diverse populations.

\section{CONFLICTS OF INTEREST}

No potential conflict of interest relevant to this article was reported.

\section{AUTHOR CONTRIBUTIONS}

Conception or design: E.J.R., S.R., W.Y.L. Acquisition, analysis, or interpretation of data: C.H.J., E.J.R., Y.C., S.R., W.Y.L. Drafting the work or revising: C.H.J., E.J.R. Final approval of the manuscript: H.K., S.R., W.Y.L.

\section{ORCID}

Chan-Hee Jung https://orcid.org/0000-0001-8988-0187

Eun-Jung Rhee https://orcid.org/0000-0002-6108-7758

Seungho Ryu https://orcid.org/0000-0002-3927-8646

Won-Young Lee https://orcid.org/0000-0002-1082-7592

\section{REFERENCES}

1. Chalasani N, Younossi Z, Lavine JE, Diehl AM, Brunt EM, Cusi K, et al. The diagnosis and management of non-alcoholic fatty liver disease: practice guideline by the American Gastroenterological Association, American Association for the Study of Liver Diseases, and American College of Gastroenterology. Gastroenterology 2012;142:1592-609.

2. Lee YH, Cho Y, Lee BW, Park CY, Lee DH, Cha BS, et al. Nonalcoholic fatty liver disease in diabetes. Part I: epidemiology and diagnosis. Diabetes Metab J 2019;43:31-45.

3. Targher G, Day CP, Bonora E. Risk of cardiovascular disease in patients with nonalcoholic fatty liver disease. N Engl J Med 2010;363:1341-50.
4. Pinzani M. Pathophysiology of liver fibrosis. Dig Dis 2015; 33:492-7.

5. Ekstedt M, Hagstrom H, Nasr P, Fredrikson M, Stal P, Kechagias $\mathrm{S}$, et al. Fibrosis stage is the strongest predictor for disease-specific mortality in NAFLD after up to 33 years of follow-up. Hepatology 2015;61:1547-54.

6. Angulo P, Kleiner DE, Dam-Larsen S, Adams LA, Bjornsson ES, Charatcharoenwitthaya $\mathrm{P}$, et al. Liver fibrosis, but no other histologic features, is associated with long-term outcomes of patients with nonalcoholic fatty liver disease. Gastroenterology 2015;149:389-97.

7. Li L, Liu DW, Yan HY, Wang ZY, Zhao SH, Wang B. Obesity is an independent risk factor for non-alcoholic fatty liver disease: evidence from a meta-analysis of 21 cohort studies. Obes Rev 2016;17:510-9.

8. Sookoian S, Pirola CJ. Systematic review with meta-analysis: the significance of histological disease severity in lean patients with nonalcoholic fatty liver disease. Aliment Pharmacol Ther 2018;47:16-25.

9. Janghorbani M, Salamat MR, Aminorroaya A, Amini M. Utility of the visceral adiposity index and hypertriglyceridemic waist phenotype for predicting incident hypertension. Endocrinol Metab (Seoul) 2017;32:221-9.

10. Gentile CL, Weir TL, Cox-York KA, Wei Y, Wang D, Reese $\mathrm{L}$, et al. The role of visceral and subcutaneous adipose tissue fatty acid composition in liver pathophysiology associated with NAFLD. Adipocyte 2015;4:101-12.

11. Park BJ, Kim YJ, Kim DH, Kim W, Jung YJ, Yoon JH, et al. Visceral adipose tissue area is an independent risk factor for hepatic steatosis. J Gastroenterol Hepatol 2008;23:900-7.

12. Van der Poorten D, Milner KL, Hui J, Hodge A, Trenell MI, Kench JG, et al. Visceral fat: a key mediator of steatohepatitis in metabolic liver disease. Hepatology 2008;48:449-57.

13. Narumi H, Yoshida K, Hashimoto N, Umehara I, Funabashi N, Yoshida S, et al. Increased subcutaneous fat accumulation has a protective role against subclinical atherosclerosis in asymptomatic subjects undergoing general health screening. Int J Cardiol 2009; 135:150-5.

14. Kim D, Chung GE, Kwak MS, Seo HB, Kang JH, Kim W, et al. Body fat distribution and risk of incident and regressed nonalcoholic fatty liver disease. Clin Gastroenterol Hepatol 2016;14:132-8.

15. Oh YH, Moon JH, Kim HJ, Kong MH. Visceral-to-subcutaneous fat ratio as a predictor of the multiple metabolic risk factors for subjects with normal waist circumference in Korea. Diabetes Metab Syndr Obes 2017;10:505-11.

Copyright (C) 2020 Korean Endocrine Society 
16. Higuchi S, Kabeya Y, Kato K. Visceral-to-subcutaneous fat ratio is independently related to small and large cerebrovascular lesions even in healthy subjects. Atherosclerosis 2017; 259:41-5.

17. Fukuda T, Bouchi R, Takeuchi T, Nakano Y, Murakami M, Minami I, et al. Ratio of visceral-to-subcutaneous fat area predicts cardiovascular events in patients with type 2 diabetes. J Diabetes Investig 2018;9:396-402.

18. Wang Y, Chen F, Wang J, Wang T, Zhang J, Han Q, et al. The relationship between increased ratio of visceral-to-subcutaneous fat area and renal outcome in Chinese adults with type 2 diabetes and diabetic kidney disease. Can J Diabetes 2019;43:415-20.

19. Pagadala MR, McCullough AJ. Non-alcoholic fatty liver disease and obesity: not all about body mass index. Am J Gastroenterol 2012;107:1859-61.

20. Feng RN, Du SS, Wang C, Li YC, Liu LY, Guo FC, et al. Lean-non-alcoholic fatty liver disease increases risk for metabolic disorders in a normal weight Chinese population. World J Gastroenterol 2014;20:17932-40.

21. Quinn SF, Gosink BB. Characteristic sonographic signs of hepatic fatty infiltration. AJR Am J Roentgenol 1985;145: 753-5.

22. Kim JA, Choi CJ, Yum KS. Cut-off values of visceral fat area and waist circumference: diagnostic criteria for abdominal obesity in a Korean population. J Korean Med Sci 2006; 21:1048-53.

23. Cheah MC, McCullough AJ, Goh GB. Current modalities of fibrosis assessment in non-alcoholic fatty liver disease. $\mathrm{J}$ Clin Transl Hepatol 2017;5:261-71.

24. Shah AG, Lydecker A, Murray K, Tetri BN, Contos MJ, Sanyal AJ, et al. Comparison of noninvasive markers of fibrosis in patients with nonalcoholic fatty liver disease. Clin Gastroenterol Hepatol 2009; 7:1104-12.

25. Wai CT, Greenson JK, Fontana RJ, Kalbfleisch JD, Marrero JA, Conjeevaram HS, et al. A simple noninvasive index can predict both significant fibrosis and cirrhosis in patients with chronic hepatitis C. Hepatology 2003;38:518-26.

26. Adams LA, George J, Bugianesi E, Rossi E, De Boer WB, van der Poorten D, et al. Complex non-invasive fibrosis models are more accurate than simple models in non-alcoholic fatty liver disease. J Gastroenterol Hepatol 2011;26: 1536-43.

27. Matthews DR, Hosker JP, Rudenski AS, Naylor BA, Treacher DF, Turner RC. Homeostasis model assessment: insulin resistance and beta-cell function from fasting plasma glucose and insulin concentrations in man. Diabetologia 1985;28:412-9.

28. Ahn Y, Kwon E, Shim JE, Park MK, Joo Y, Kimm K, et al. Validation and reproducibility of food frequency questionnaire for Korean genome epidemiologic study. Eur J Clin Nutr 2007;61:1435-41.

29. American Diabetes Association. 2. Classification and diagnosis of diabetes: standards of medical care in diabetes-2019. Diabetes Care 2019 Jan;42(Suppl 1):S13-28.

30. Angulo P, Machado MV, Diehl AM. Fibrosis in nonalcoholic fatty liver disease: mechanisms and clinical implications. Semin Liver Dis 2015;35:132-45.

31. Boutari C, Perakakis N, Mantzoros CS. Association of adipokines with development and progression of nonalcoholic fatty liver disease. Endocrinol Metab (Seoul) 2018;33:3343.

32. Moon YA. The SCAP/SREBP pathway: a mediator of hepatic steatosis. Endocrinol Metab (Seoul) 2017;32:6-10.

33. Neuschwander-Tetri BA. Hepatic lipotoxicity and the pathogenesis of nonalcoholic steatohepatitis: the central role of nontriglyceride fatty acid metabolites. Hepatology 2010;52: 774-88.

34. Fan JG, Kim SU, Wong VW. New trends on obesity and NAFLD in Asia. J Hepatol 2017;67:862-73.

35. Younossi ZM, Stepanova M, Negro F, Hallaji S, Younossi Y, Lam B, et al. Nonalcoholic fatty liver disease in lean individuals in the United States. Medicine (Baltimore) 2012;91: 319-27.

36. Kim Y, Chang Y, Cho YK, Ahn J, Shin H, Ryu S. Obesity and weight gain are associated with progression of fibrosis in patients with nonalcoholic fatty liver disease. Clin Gastroenterol Hepatol 2019;17:543-50.

37. Sumida Y, Nakajima A, Itoh Y. Limitations of liver biopsy and non-invasive diagnostic tests for the diagnosis of nonalcoholic fatty liver disease/nonalcoholic steatohepatitis. World J Gastroenterol 2014;20:475-85.

38. Vallet-Pichard A, Mallet V, Nalpas B, Verkarre V, Nalpas A, Dhalluin-Venier V, et al. FIB-4: an inexpensive and accurate marker of fibrosis in $\mathrm{HCV}$ infection: comparison with liver biopsy and fibrotest. Hepatology 2007;46:32-6.

39. Kruger FC, Daniels CR, Kidd M, Swart G, Brundyn K, van Rensburg C, et al. APRI: a simple bedside marker for advanced fibrosis that can avoid liver biopsy in patients with NAFLD/NASH. S Afr Med J 2011;101:477-80.

40. Angulo P, Bugianesi E, Bjornsson ES, Charatcharoenwitthaya P, Mills PR, Barrera F, et al. Simple noninvasive sys- 
tems predict long-term outcomes of patients with nonalcoholic fatty liver disease. Gastroenterology 2013;145:782-9. 41. Kim D, Kim WR, Kim HJ, Therneau TM. Association be- tween noninvasive fibrosis markers and mortality among adults with nonalcoholic fatty liver disease in the United States. Hepatology 2013;57:1357-65. 